Europhys. Lett., 61 (3), pp. 415-421 (2003)

\title{
Dynamical instability in driven colloids
}

\author{
J. Chakrabarti ${ }^{1}$, J. Dzubiella ${ }^{2}$ and H. Löwen ${ }^{2}$ \\ 1 S. N. Bose National Centre for Basic Sciences, Block-JD, Sector-III \\ Salt Lake, Calcutta, 700091, India \\ 2 Institut für Theoretische Physik II, Heinrich-Heine-Universität Düsseldorf \\ Universitätsstraße 1, D-40225 Düsseldorf, Germany
}

(received 17 May 2002; accepted in final form 25 November 2002)

PACS. 82.70.-y - Disperse systems; complex fluids.

PACS. 61.20.-p - Structure of liquids.

PACS. 05.40.-a - Fluctuation phenomena, random processes, noise, and Brownian motion.

\begin{abstract}
Brownian-dynamics computer simulations show a dynamical crossover in a strongly interacting colloidal suspension consisting of oppositely driven particles, wherein a uniform state transforms, with increasing driving force, into a locally demixed state characterized by strongly anisotropic stripe-like domains which are homogeneous in the direction parallel to the drive but have finite spatial extent of a double-correlation length in the transverse direction. A phenomenological dynamic density-functional theory has been proposed which accounts for such a strongly anisotropic state as arising from an instability of a homogeneous state.
\end{abstract}

Introduction. - Nonequilibrium phase transitions are ubiquitous in nature, since they occur in simple and complex fluids [1-3], in granular matter [4], in enormously complicated biological systems $[5,6]$ as well as in pedestrian dynamics [7]. In order to understand the principles of such phase transformations, simple lattice models of driven diffusive systems have been studied $[4,8,9]$ extensively in the recent past. However, most of the realizations in nature are off-lattice, implying a much richer scenario of nonequilibrium transitions. Here, phenomenological treatments have had considerable success [1], but full microscopic theories involving the interparticle interactions as the only input are still missing.

Mesoscopic colloidal suspensions [10] represent excellent realizations of driven diffusive systems with a number of advantages: The samples are well characterized and the effective particle interactions can be tailored. Moreover, external driving fields can be applied in a controlled way [11]. The mesoscopic length scales and the slow relaxation times of the order of milliseconds open the way for a direct experimental investigation in real space and real time which is impossible for molecular systems. For instance, colloidal samples can be confined between glass plates to two dimensions [12] such that their individual trajectories can be followed by video microscopy [13]. For equilibrium phase transformations, colloids have played an explicit role as model systems for condensed matter in general, and it can be expected that they will play a similarly dominant role for nonequilibrium phase transitions.

In this paper we examine a particular kind of nonequilibrium segregation in a driven two-component colloidal mixture by theory and simulation. In our two-dimensional model, the two components are driven in opposite directions by constant external forces. Using Brownian-dynamics simulations, we observe a dynamical crossover upon increasing the driving force. Above a critical drive, the system proceeds from a uniform mixed fluid into a state 
characterized by strongly anisotropic stripe-like domains in the direction parallel to the driving field. We further show that this phenomenon can be understood from a dynamical densityfunctional theory that takes the repulsive interparticle interaction explicitly into account. The dynamical segregation is triggered by the interparticle interactions and only occurs in strongly interacting and overdamped Brownian fluids, even without any hydrodynamic interactions. It is therefore qualitatively different from previous studies of segregation transitions which are either generated by hydrodynamic flow [14-16] or by a finite inertia of the particles as relevant in granular matter $[17,18]$ and pedestrian models [7]. Reference [3] reports Brownian-dynamics computer simulations on a similar system where first-order laning transition is observed for very strong driving force, the extent of the segregated stripes perpendicular to the drive being macroscopic, i.e., comparable to the system size. Here we focus on the low driving-force regime in order to have a better understanding of the mechanism of such lane formation. In what follows we first describe results from computer simulations of our model. Then we construct a dynamical density-functional theory to account for the stability of the inhomogeneous phase.

Brownian computer simulation. - We simulate $2 N=500$ colloidal particles in a square cell of length $\ell$, having periodic boundary conditions with a fixed number density $\rho_{0}=2 \mathrm{~N} / \ell^{2}$ and at a fixed temperature $T$. The effective pair potential between two colloidal particles at a separation $r$ is modeled as a screened Coulomb interaction [10]

$$
V(r)=V_{0} \sigma \exp [-\kappa(r-\sigma) / \sigma] / r
$$

where $V_{0}$ is an energy scale, $\sigma$ is the length scale, defining the range of the interaction, and $\kappa$ the reduced inverse screening length. In this work the energy is chosen to be $V_{0}=10 k_{\mathrm{B}} T$, where $k_{\mathrm{B}} T$ is the thermal energy, and $\kappa=4.0$ is fixed.

The dynamics of the colloids is completely overdamped Brownian motion. We neglect hydrodynamic interactions which is justified for small colloidal volume fractions. We solve numerically, using the technique of Ermak $[19,20]$ over a time-step $\Delta t$, the stochastic Langevin equations for the colloidal trajectories $\vec{r}_{i}(t)(i=1, \ldots, 2 N)$ :

$$
\gamma \frac{\mathrm{d} \vec{r}_{i}}{\mathrm{~d} t}=-\vec{\nabla}_{\vec{r}_{i}} \sum_{j \neq i} V\left(\left|\vec{r}_{i}-\vec{r}_{j}\right|\right)+F_{i} \vec{e}_{y}+\vec{F}_{i}^{(\mathrm{R})}(t) .
$$

There are different forces acting onto the colloidal particles: The first term is the force attributed to inter-particle interactions. Next is the constant external field acting in the $y$ direction of the simulation cell. The particles are oppositely driven: $F_{i}=F>0$ for $(+)$ particles and $F_{i}=-F$ for $(-)$ particles. Half of the particles are of type $(+)$, the other half is of type $(-)$ such that their partial number densities are $\rho_{+}^{0}=\rho_{-}^{0}=\rho_{0} / 2$. We denote the dimensionless drive parameter by $F^{*}=F \sigma / k_{\mathrm{B}} T$. Finally, random forces $\vec{F}_{i}^{(\mathrm{R})}$ describe the kicks of the solvent molecules acting onto the $i$-th colloidal particle. These kicks are Gaussian random numbers with zero mean, $\overrightarrow{\vec{F}_{i}^{(\mathrm{R})}}=0$, and variance

$$
\overline{\left(\vec{F}_{i}^{(\mathrm{R})}\right)_{\alpha}(t)\left(\vec{F}_{j}^{(\mathrm{R})}\right)_{\beta}\left(t^{\prime}\right)}=2 k_{\mathrm{B}} T \gamma \delta_{\alpha \beta} \delta_{i j} \delta\left(t-t^{\prime}\right) .
$$

The subscripts $\alpha$ and $\beta$ stand for the two Cartesian components. The friction constant $\gamma$ is assumed to be the same for both types of particles. We choose $\Delta t=0.003 \tau_{\mathrm{B}}$, where $\tau_{\mathrm{B}}=\gamma \sigma^{2} / V_{0}$ is a typical Brownian timescale. After an initial relaxation period of $20 \tau_{\mathrm{B}}$, the steady-state statistics has been gathered. In order to detect anisotropic correlations, we measure the steady-state structure factors of a given species $S_{k}\left(q_{k}\right)$ in the $k=x, y$ direction 
a)

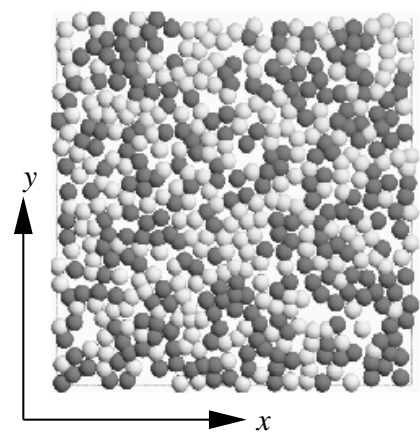

b)

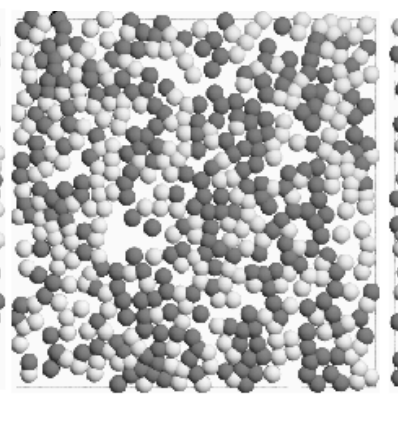

c)

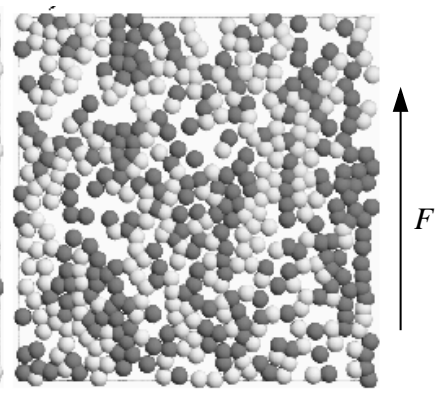

Fig. 1 - Simulation snapshots for different external forces: a) $F^{*}=1.0$, b) $F^{*}=6.0$, c) $F^{*}=10.0$. The particles are rendered as spheres with diameters $\sigma$. (+)-particles are dark gray, while (-)-particles are light gray. The arrows indicate the $x$ - and $y$-directions.

from the steady-state configurations:

$$
S_{k}\left(q_{k}\right)=\left\langle\frac{1}{\mathcal{N}} \rho\left(q_{k}\right) \rho\left(-q_{k}\right)\right\rangle
$$

with $\rho\left(q_{k}\right)=\sum_{j=1}^{\mathcal{N}} \exp \left[\mathrm{i} q_{k} k_{j}\right]$, where $k_{i}=x_{i}, y_{i}$ are the $x$ and $y$ coordinates of the $i$-th particle. Here, two particles of the same species are considered for averaging if their distance projected to the perpendicular direction of $k=x, y$ is smaller than a suitable length $s$. If $s$ is too small, the finite time of the simulation allows no good statistics. If $s$ is too large, all correlations in the $k$-direction will vanish on average. We find that a suitable choice is $s=5 \rho_{0}^{-1 / 2}$. We have checked carefully that the choice of $s$ has no influence on the qualitative behavior of the structure factors, except a slight shift in the peak heights. The brackets $\langle\cdots\rangle$ in eq. (4) denote averaging over time, all slabs with width $s$ and the particle types. $\mathcal{N}$ is the average number of particles of the considered type contained in one of these slabs serving as a normalization factor.

We report in particular observations for $\rho_{0} \sigma^{2}=0.3$. The snapshot in fig. 1a) shows that the two species $[+,-]$ remain mixed in a homogeneous state for low $F^{*}=1.0$. However, around a critical drive, $F^{*}=6.0$, the system tends to get into a highly anisotropic structure, shown in fig. 1b): droplets of similarly driven particles, highly elongated along $y$ and having characteristic transverse dimension $\sim 1 / q_{0}$, are formed. This gets much more pronounced for higher drivings, see fig. 1c). The lanes of similarly driven particles proliferate parallel to the $y$-direction. These observations are supported by $S_{x}\left(q_{x}\right)$ and $S_{y}\left(q_{y}\right)$ data as well. $S_{x}\left(q_{x}\right)$ in fig. 2a) shows a growing low $q_{x}$ peak beyond $F^{*}=6.0$ roughly at half of the correlation peak maximum implying that the substructure is a doubled-correlation length in the $x$-direction.

The continuous growth of the peak at $q_{x}^{*} \approx 1$ of $S_{x}\left(q_{x}\right)$ of our simulation data signals the onset of strongly anisotropic spatial correlations. The onset seems to be a sharp crossover between a spatially homogeneous system and a system with a "worm-like" structure of strongly anisotropic spatial correlations. The low peak slightly shifts to lower $q_{x}$ as $F^{*}$ increases. The substantial homogenization in the $y$-direction, characterized by the growth of a $q_{y}=0$ peak, is observable in the structure factor $S_{y}\left(q_{y}\right)$ in fig. $\left.2 \mathrm{~b}\right)$. The simulations are thus suggestive of structural inhomogeneity being supported in the system above a critical $F^{*}$. The critical field $F_{\mathrm{c}}^{*}$ is estimated by the $F^{*}$ where the low $q_{x}$ peak in $S_{x}\left(q_{x}\right)$, shown in fig. 2a), first appears. A 


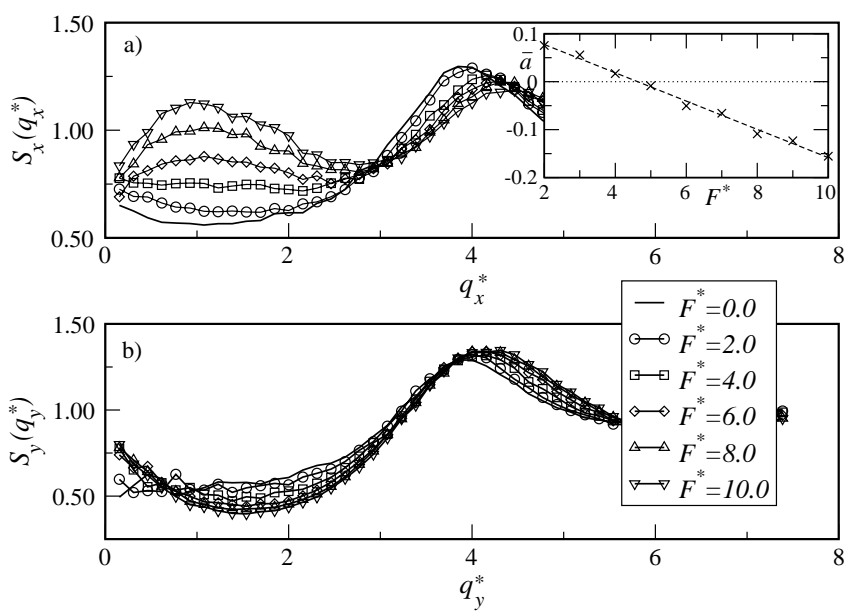

Fig. 2 - Structure factors $S_{x}\left(q_{x}\right)$ and $S_{y}\left(q_{y}\right)$ as defined in eq. (4) for different external forces for a density $\rho_{0} \sigma^{2}=0.3$. The inset in a) shows the curvature $a$ (crosses) of a parabolic fit to $S_{x}\left(q_{x}\right)$ in an interval $q_{x}^{*} \in[0 \ldots 3]$. The zero point of the linear fit (dashed line) defines the critical force, which is $F_{\mathrm{c}}^{*}=4.7$ for this particular density.

parabolic function $\bar{a} q_{x}^{2}+\bar{b} q_{x}+\bar{c}$, with $\bar{a}, \bar{b}, \bar{c}$ as the parameters, is fitted to the data of $S_{x}\left(q_{x}\right)$ in the interval $q_{x}^{*} \in[0,3]$. For $F<F_{\mathrm{c}}$ the curvature $\bar{a}$ is positive, while for $F^{*}>F_{\mathrm{c}}$ the peak has a negative curvature. Hence, the zero of the curvature in the parabolic fit is a reliable estimate for the onset of anisotropic spatial correlations. An example of the curvature $\bar{a} v s$. the force results is shown in the inset of fig. $2 \mathrm{a}$ ) for a density $\rho \sigma^{2}=0.3$. We remark that the critical forces obtained for anisotropic coarsening are significantly smaller than those required for the full first-order lane formation as obtained in ref. [3]. Thus the crossover found here is a precursor to the lane formation [3], the latter being preceded by strongly anisotropic domain formation at much lower $F^{*}$.

Dynamical density-functional theory. - We now develop a dynamical density-functional theory to account for the stabilization of the inhomogeneous steady states beyond a critical drive strength as suggested by the simulations. We start from the continuity equation of the inhomogeneous time-dependent density fields $\rho_{ \pm}(\vec{r}, t)$ :

$$
\frac{\partial \rho_{ \pm}(\vec{r}, t)}{\partial t}=-\nabla \cdot\left[\vec{j}_{ \pm}^{(1)}(\vec{r}, t)+\vec{j}_{ \pm}^{(2)}(\vec{r}, t)+\vec{j}_{ \pm}^{(3)}(\vec{r}, t)\right]
$$

where we include three different types of currents: $\vec{j}_{ \pm}^{(k)}(\vec{r}, t)(k=1,2,3)$. The first term, $\vec{j}_{ \pm}^{(1)}(\vec{r}, t)$, is a diffusive current generated by a chemical potential gradient in the system. In the framework of dynamical density-functional theory it is modeled as [21]

$$
\vec{j}_{ \pm}^{(1)}(\vec{r}, t)=-\frac{D}{k_{\mathrm{B}} T} \rho_{ \pm}(\vec{r}, t) \nabla \frac{\delta \mathcal{F}\left[\rho_{+}, \rho_{-}\right]}{\delta \rho_{ \pm}(\vec{r}, t)},
$$

where $D=k_{\mathrm{B}} T / \gamma$ is the diffusion coefficient. The free-energy density-functional, $\mathcal{F}\left[\rho_{+}, \rho_{-}\right]$, for a strongly correlated system can be expressed for weak inhomogeneities around a homogeneous state as [22]

$$
\frac{\mathcal{F}\left[\rho_{+}, \rho_{-}\right]}{k_{\mathrm{B}} T}=\sum_{k= \pm} \int \mathrm{d}^{2} r \rho_{k}(\vec{r}) \log \left[2 \rho_{k}(\vec{r}) / \rho_{0}\right]-\frac{1}{2} \int \mathrm{d}^{2} r \mathrm{~d}^{2} r^{\prime} c\left(\left|\vec{r}-\vec{r}^{\prime}\right|\right) \Delta \rho(\vec{r}) \Delta \rho\left(\overrightarrow{r^{\prime}}\right)
$$


with $\Delta \rho(\vec{r})=\rho_{+}(\vec{r})+\rho_{-}(\vec{r})-\rho_{0}$. Here $c(r)$ is the fluid direct correlation function, determined by the interparticle interactions $V(r)[22]$. The second type of current, $\vec{j}_{ \pm}^{(2)}(\vec{r}, t)$, is directly induced by the driving field in the $y$-direction. In a completely demixed state, we know that the field induces a Brownian drift velocity of $v_{\mathrm{d}}=F / \gamma$, while in a completely mixed state the flow is reduced due to mutual collisions between oppositely driven particles. Assuming that there is no drift at all in the latter case and interpolating linearly between demixed and mixed situations we obtain $\left({ }^{1}\right)$

$$
\vec{j}_{ \pm}^{(2)}(\vec{r}, t)=\mp \vec{e}_{y} \frac{2 v_{\mathrm{d}}}{\rho_{0}}\left[\rho_{+}(\vec{r}, t)\left(\rho_{0} / 2-\rho_{+}(\vec{r}, t)\right)-\rho_{-}(\vec{r}, t)\left(\rho_{0} / 2-\rho_{-}(\vec{r}, t)\right)\right] .
$$

Finally, the third type of current, $\vec{j}_{ \pm}^{(3)}(\vec{r}, t)$, is also induced by the external drive, but it is in the $x$-direction perpendicular to the field. The physics of this term can be understood by Brownian collisions between + and - particles causing a displacement perpendicular to the field. Were the difference of the density fields $\rho_{+}(\vec{r}, t)-\rho_{-}(\vec{r}, t)$ homogeneous in the $x$ direction, there would not be any net effect on average, so that $\vec{j}_{ \pm}^{(3)}$ would vanish. Hence, the first nontrivial leading term contributing to $\vec{j}_{ \pm}^{(3)}(\vec{r}, t)$ is the gradient in the density difference field. Furthermore, $\vec{j}_{ \pm}^{(3)}(\vec{r}, t)$ should scale with the local collision number per time. With $\sigma_{0}$ denoting a typical range of the interaction $V(r)$ needed to perform a collision, the latter quantity is proportional to $v_{\mathrm{d}} \sigma_{0} \rho_{+}(\vec{r}, t) \rho_{-}(\vec{r}, t)$. Consequently, we find

$$
\vec{j}_{ \pm}^{(3)}(\vec{r}, t)= \pm \vec{e}_{x} \frac{\sigma_{0} v_{\mathrm{d}}}{\rho_{+} \rho_{-}} \rho_{+}(\vec{r}, t) \rho_{-}(\vec{r}, t) \frac{\partial}{\partial x}\left[\rho_{+}(\vec{r}, t)-\rho_{-}(\vec{r}, t)\right],
$$

where the factor $1 /\left(\rho_{+} \rho_{-}\right)$serves to guarantee correct dimensions. Henceforth, we estimate $\sigma_{0}$ to be an effective hard-core diameter from the Barker-Henderson perturbation theory, $\sigma_{0}=\int_{0}^{\infty}\left[1-\exp \left[-V(r) / k_{\mathrm{B}} T\right]\right] \mathrm{d} r$. We finally remark that — as a symmetry requirement- all currents remain unchanged under the interchange of the species, namely, $\rho_{+}(\vec{r}, t) \leftrightarrow \rho_{-}(\vec{r}, t)$ and $F \rightarrow-F$.

A linear stability analysis for density perturbations in the steady state [2], proportional to $\exp \left[i q_{x} x+i q_{y} y+\omega t\right]$, yields two possible dispersion relations:

$$
\begin{aligned}
\omega_{1}^{*} & =2 F^{*} q_{x}^{2}-\left[1-\rho_{0} \tilde{c}(\vec{q})\right] q^{2}-6 i v_{\mathrm{d}} \sigma_{0} q_{y} / D, \\
\omega_{2}^{*} & =-\left[1-\rho_{0} \tilde{c}(\vec{q})\right] q^{2}
\end{aligned}
$$

where $\omega_{1,2}^{*}=\omega_{1,2} \sigma_{0}^{2} / D$ denotes the dimensionless frequency and $\tilde{c}(\vec{q})$ is the Fourier transform of $c(r)$. Steady-state bifurcations require the frequencies to be real which can be achieved by $q_{y}=0$. Note that $1-\rho_{0} \tilde{c}(\vec{q})$, being the inverse of the static structure factor, is a positivedefinite quantity [22]. Hence, $\omega_{2}^{*}$ remains negative for all $\vec{q}=\left(q_{x}, q_{y}\right)$. The real $\omega_{1}^{*}$ for $q_{y}=0$ can change sign by virtue of the competition of two terms, indicating the steady-state bifurcation of a homogeneous state to an inhomogeneous state [2]. Physically, the competition is between the diffusive thermodynamic current which - via entropy of mixing - tries to avoid any +- interface and the current $\vec{j}_{ \pm}^{(3)}(\vec{r}, t)$ which amplifies particle segregation in the $x$-direction via collisions induced by the drive. Being the unstable wave vector in the $y$ direction $q_{y}=0$ as in the simulations, the $q_{x}$ where $\omega_{1}^{*}$ has a positive maximum will dominate the growth of the inhomogeneous phase. The role of the correlations in stabilizing the patterns

$\left({ }^{1}\right)$ If a term $v_{\mathrm{d}} / \rho_{0} \alpha \overrightarrow{e_{y}} \rho_{+}(r, t) \rho_{-}(r, t)$ with small $\alpha$ is added to the r.h.s. of eq. (8) in order to account for nonzero flow in the mixed states, the requirement $q_{y}=0$ for real $w_{1,2}$ still remains and the results are unaffected. 

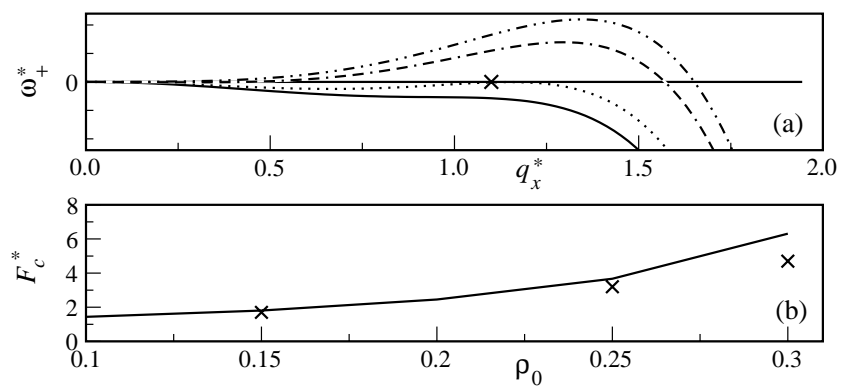

Fig. 3 - a) Dispersion curves for a density $\rho_{0} \sigma^{2}=0.3$ and forces $F^{*}=6.25,6.75,7.2$ and 7.75. The curves move up with increasing external drive. The cross denotes the location of $q_{0}$ corresponding to the critical force $F^{*}=4.7$ obtained from simulation. b) Critical drive $F_{\mathrm{c}}^{*} v s$. density $\rho_{0} \sigma^{2}$. The theoretical result (solid line) is compared to simulation results (crosses).

is apparent from the dispersion relations. In the high-temperature limit towards the ideal gas, all correlations vanish. Then $\omega_{1}^{*}$ does not show any maximum for a finite $q_{x}$. Thus, the structural inhomogeneity, characterized by a small and finite $q_{0}$ in the $x$-direction, can be supported only in a strongly correlated system.

In order to locate the unstable low- $q_{x}$ modes as the steady-state bifurcation takes place for the finite- $\rho_{0}$ cases, we approximate $c(r)$ by that of an effective hard-disk fluid with effective diameter $\sigma_{0}$ for which analytical expressions are known [23]. The behavior of $\omega_{1}^{*}$ as a function of $q_{x}$ is shown in fig. 3a) for a given $\rho_{0}$ and some typical values of $F^{*}$ : For very low $F^{*}$, $\omega_{1}^{*}$, despite having a maximum at a nontrivial $q_{x}$, remains negative for all $q_{x}$ indicating the stability of the homogeneous phase. As $F^{*}$ increases, the maximum at the nontrivial $q_{x}$, $q_{0}$, touches the zero axis at $F^{*}=F_{\mathrm{c}}^{*}$, indicating that a mode with wave vector $q_{0}$ becomes marginally stable. The homogeneous phase is unstable for $F^{*}>F_{\mathrm{c}}$ to density perturbations with $q_{x}$ over a band of about $q_{0}$, the growth being dominated by $q_{0}$. We also observe from fig. 3a) that both $q_{0}$ and $\omega_{1}^{*}\left(q_{0}\right)$ increase with $F^{*}$ for $F^{*}>F_{\mathrm{c}}^{*}$. We determine graphically $F_{\mathrm{c}}^{*}$ as a function of $\rho_{0}$, as shown by the solid line in fig. $\left.3 \mathrm{~b}\right)$. We note that $F_{\mathrm{c}}^{*}$ increases with $\rho_{0}$. The critical fields $F_{c}^{*}$ for the crossover in the simulations, marked by the crosses in fig. $3 \mathrm{~b}$ ), also show a very similar trend. Moreover, the increase in $\omega_{1}^{*}\left(q_{0}\right)$ for $F^{*}>F_{\mathrm{c}}^{*}$ is in qualitative agreement with the growth of the low $q_{x}$ peak in $S_{x}\left(q_{x}\right)$ for $F^{*}>F_{c}^{*}$ in the simulations. We further observe that $q_{0}$ agrees well with the low $q_{x}$ peak position of $S_{x}\left(q_{x}\right)$ at the simulation estimate of $F_{c}^{*}$ which corresponds to a double-correlation length. The simulation data show a shift of the low $q_{x}$ peak to higher values within the noise, for $F^{*}>F_{\mathrm{c}}^{*}$, as in the theory. Such similarities in the features lead us to link the dynamical instability found in our theory to the onset of strongly anisotropic spatial correlations in the simulations.

Conclusions. - We summarize our findings: An equimolar mixture of oppositely driven and strongly interacting colloidal particles proceeds from a homogeneous state to a stripepatterned state characterized by strongly anisotropic correlations. The stripes are homogeneous in the direction parallel to the drive but have finite spatial extent of a doubled-correlation length in the transverse direction. Our dynamical density-functional theory yields the dynamical instability as a steady-state bifurcation of the density field around the onset of structural inhomogeneity in the simulations. This suggests the following mechanism of lane formation of ref. [3]: The homogeneous state becomes unstable to inhomogeneities with anisotropic domains via a steady-state bifurcation. Such domains proliferate to form lanes for very large drive where nonlinearities could be important. Further works would be necessary to verify this 
conjecture. Qualitatively, we expect that the instability persists for asymmetric mixtures, for different repulsive interactions $V(r)$, for slowly oscillating external fields and for three spatial dimensions. Our prediction can be verified in real-space experiments of highly charged and strongly deionized colloidal mixtures, where the colloidal volume fraction is small so that hydrodynamic interactions do not play a dominant role. If the particles have the same charge but a different mass, one should see the instability during sedimentation. Another realization is an electric (or magnetic) field acting on a bidisperse charged mixture (with magnetizable cores). We finally would like to point out that the dynamical density-functional theory presented here to include explicitly the effect of particle interaction in a driven system should be useful in understanding dynamical instabilities encountered in other systems, as, e.g., pedestrian dynamics [24].

$$
* * *
$$

We thank J. Sherwood, B. Schmittmann and H. K. Janssen for helpful discussions and the DFG for support within the SFB 237.

\section{REFERENCES}

[1] Cross M. C. and Hohenberg P. C., Rev. Mod. Phys., 65 (1993) 851.

[2] Nicolis G. and Prigogine I., Self-organization in Nonequilibrium Chemical Systems (SpringerVerlag, Berlin) 1977.

[3] Dzubiella J. et al., Phys. Rev. E, 65 (2002) 021402.

[4] Sides S. W. et al., Phys. Rev. Lett., 81 (1998) 834.

[5] Koch A. J. and Meinhardt H., Rev. Mod. Phys., 1481 (1994) 66.

[6] Murray J. D., Mathematical Biology (Springer, Berlin) 1990.

[7] Helbing D. et al., Phys. Rev. Lett., 84 (2000) 1240.

[8] Schmittmann B. and Zia R. K. P., in Phase Transitions and Critical Phenomena, edited by Domb C. and Lebowitz J., Vol. 17 (Academic Press, London) 1995.

[9] Chakrabarti B. K. and Acharyya M., Rev. Mod. Phys., 71 (1999) 847.

[10] Pusey P. N., in Liquids, Freezing and the Glass Transition, edited by Hansen J. P. et al. (North Holland, Amsterdam) 1991.

[11] Dhont J. K. G., An Introduction to Dynamics of Colloids (Elsevier, Amsterdam) 1996.

[12] Wei Q. H. et al., Phys. Rev. Lett., 81 (1998) 2606.

[13] Murray C. A. and Grier D. G., Annu. Rev. Phys. Chem., 47 (1996) 421.

[14] Whitmore R. L., Brit. J. Appl. Phys., 6 (1955) 239.

[15] Batchelor G. K. and Janse van Rensburg R. W., J. Fluid. Mech., 166 (1986) 379.

[16] Cox R. G., Int. J. Multiphase Flow., 16 (1990) 617.

[17] Valiveti P. and Косh D. L., Phys. Fluids, 11 (1999) 3283.

[18] Santra S. B. et al., Phys. Rev. E, 54 (1996) 5066.

[19] Allen M. P. and Tildesley D. J., Computer Simulations of Liquids (Clarendon Press, Oxford) 1989.

[20] Ermak D. L., J. Chem. Phys., 62 (1975) 4189.

[21] Chaikin P. M. and Lubensky T. C., Principles of Condensed Matter Physics (Cambridge University Press, Cambridge) 1995.

[22] Hansen J. P. and McDonald I. R., Theory of Simple Liquids, 2nd edition (Academic Press, London) 1986.

[23] Baus M. and Colot J.-L., Phys. Rev. A, 36 (1987) 3912.

[24] Helbing D. et al., Environment and Planning B: Planning and Design, 28 (2001) 361. 